\title{
Is the Stellar System WR 11 a Gamma-Ray Source?
}

\author{
Paula Benaglia ${ }^{1,2,3}$ \\ ${ }^{1}$ Instituto Argentino de Radioastronomía, CCT-La Plata, CONICET, CC5 (1894) Villa Elisa, Buenos Aires, Argentina \\ ${ }^{2}$ Facultad de Ciencias Astronmicas y Geofsicas, UNLP, Paseo del Bosque s/n (1900), La Plata, Buenos Aires, Argentina \\ ${ }^{3}$ Email: pbenaglia@fcaglp.unlp.edu.ar
}

(ReCEIVED February 7, 2016; ACCEPTED March 24, 2016)

\begin{abstract}
Many early-type stars are in systems; some of them have been indicated as putative high-energy emitters. The radiation would be produced at the region where two stellar winds collide. Compelling evidence of such emission was found only for the colliding-wind binary (CWB) Eta Car, which was associated to a GeV source. Very recently, the closest CWB, WR 11, was proposed as a counterpart of a $6 \sigma$ emission excess, measured with the Fermi LAT satellite. We sought evidence to support or reject the hypothesis that WR 11 is responsible of the gamma-ray excess. Archive radio interferometric data at 1.4 and $2.5 \mathrm{GHz}$ taken with the Australia Telescope Compact Array along 16 dates were reduced. The sizes of the field-of-view at $2.5 \mathrm{GHz}$ and of the central region of the Fermi LAT excess are alike. We analysed the emission of the WR 11 field, characterised the radio sources detected and derived their spectral indices, to investigate their nature. Eight sources with fluxes above $10 \mathrm{mJy}$ were detected at both frequencies. All but one (WR 11) showed negative spectral indices. Four of them were identified with known objects, including WR 11. A fifth source, labeled here S6, is a promising candidate to produce gamma-ray emission, besides the CWB WR 11.
\end{abstract}

Keywords: Radio continuum: stars - stars: individual: WR 11 - stars: winds, outflows

\section{INTRODUCTION}

The first gamma-ray all-sky observations, obtained decades ago with the satellites COS-B (Hermsen 1983) and Compton (Hartman et al. 1999), disclosed numerous sources with not known counterpart at other wavelengths, hereafter called unidentified gamma-ray sources or UNIDS. Since then, a large number of multifrequency observations have been implemented to clarify the nature of those sources (Massaro et al. 2013; Paredes et al. 2008). Nowadays, though telescope capabilities have been largely expanded, like the source localisation, there still remain hundreds of gamma-ray sources to be identified. For instance, the third Fermi LAT catalogue (Acero et al. 2015, around 3000 sources, hereafter 3FGL) listed one-third of the sources with unknown counterpart at other energy range.

The identified gamma-ray sources are mostly Active Galactic Nuclei, pulsars, SNRs, and High-Mass X-ray Binaries. Many of these objects emit at radio waves, especially at low frequencies, where synchrotron radiation is stronger. This turns the radio band the most preferred to search for UNIDS counterparts.

The information collected at radio frequencies often serves to predict the behaviour of the systems in the high-energy (HE) domain, and the predictions can be compared with the observations. The same population of particles (i.e. electrons) is involved at the same time with magnetic fields, producing low energy synchrotron photons, as well as with photon fields, producing HE photons through Inverse-Compton (IC) scattering. In this way, the radio data can be used to impose severe constraints to the HE spectrum. The results will thus help to develop more complex and accurate models, leading to a fruitful iteration between theory and observations.

Some early-type (OB) stars and their descendants WolfRayet stars have been indicated as putative HE emitters (Romero, Benaglia, \& Torres 1999). If two (or more) of those stars form a stellar system, their strong winds can interact with magnetic fields and relativistic particles, in a wind-collision region, as was described by Eichler \& Usov (1993). Candidates, scenarios, and conditions under which HE can be produced are given in Dougherty \& Williams (2000), Benaglia et al. (2001), Benaglia \& Romero (2003), Reimer, Pohl, \& Reimer (2006), Pittard et al. (2006) and Reimer \& Reimer (2009). Lately, gamma-ray AGILE satellite observations allowed Tavani et al. (2009), for the first time, to present evidence of $\mathrm{HE}$ emission from the collidingwind binary (CWB) Eta Car, an LBV $+\mathrm{O}$ ? system. Additional confirming evidence using Fermi LAT data (Abdo et al. 2010) was soon published.

After compiling radio, $\mathrm{X}$-ray and gamma-ray data for $\mathrm{OB}$ and WR stars, De Becker \& Raucq (2013) presented a very 
comprehensive catalogue of particle acceleration colliding wind binaries, or PACWBs, with more than 40 candidates, and discussed the main problems related to this subject. Werner et al. (2013) modelled possible HE emission produced by WR 11 and tagged it as one of the best CWB candidates to be detected with the Fermi LAT. The latest Fermi LAT release reunited data from 2008 to 2015. Reitberger et al. (2015) derived from it an increase of the flux coming from Eta Car as the system approached periastron. Pshirkov (2016) also analysed the 7-yr Fermi LAT data, and reported emission excess coincident with the field of the nearest WR+OB system, WR 11. He ascribed the emission to the stellar system. However, the Fermi LAT excess extends over a square degree-the maximum itself covers about $10^{2}$ arcmin of the sky - encompassing hundreds of sources. And there is a significant volume of radio observations that targeted WR 11, that cover the central region of the Fermi source. I have carried out the reduction and analysis of highresolution archive radio data of the WR 11 field, in looking for additional possible counterparts to the Fermi emission. The results are presented here.

Section 2 contains the information of the system WR 11 that is relevant to this study. Section 3 describes the archive data reduction and the stellar field in the light of the radio continuum observations. Correlation results are presented in Section 4 and discussed in Section 5. In Section 6, the main conclusions are highlighted.

\section{THE STELLAR SYSTEM WR 11}

The source WR 11, also known as Gamma ${ }^{2}$ Velorum or $\gamma$ Vel, is a double-lined spectroscopic binary system, and the nearest CWB, composed by a WC8 and a O7.5 II-III stars. It is located at RA, Dec $(\mathrm{J} 2000)=8: 09: 31.95,-47: 20: 11.71$, at a distance of $\sim 370 \mathrm{pc}$ (Millour et al. 2007), and has a period of $78.5 \mathrm{~d}$ (North et al. 2007). The object was observed with the Australia Telescope Compact Array ${ }^{1}$ (ATCA) (Leitherer, Chapman, \& Koribalski 1997; Chapman et al. 1999) at four bands, from 1.4 to $8.64 \mathrm{GHz}$. The measured fluxes allowed to derive a spectral index $\alpha$ decreasing from 1.2 to 0.3 with growing frequency. (Throughout the text, we adopted the convention $S \propto v^{\alpha}$, where $S$ is the radio flux density at the frequency $v$.) Based on that, Chapman et al. (1999) stated that the emission from WR 11 could have some non-thermal (NT) contribution. The system is listed in the PACWBs catalogue, along with detailed information. At X-rays, the source is detected and the emission is explained by a hot collisional plasma, the stellar winds; the low energy photons are absorbed (Schild et al. 2004).

Optical spectro-polarimetric data of WR 11 with the ESPaDOnS Canada-France-Hawaii Telescope provided no definite detection of the magnetic field in the winds of WR 11 (de la Chevrotière et al. 2014), but a field strength upper

\footnotetext{
${ }^{1}$ The ATCA is funded by the Commonwealth of Australia for operation as a National Facility managed by CSIRO.
}

limit of $B_{\max } \sim 500 \mathrm{G}$. Data collected after the first $2 \mathrm{yr}$ by Fermi LAT were analysed (Werner et al. 2013), with negative results on a WR 11 detection. The distance from WR 11 to the closest known 3FGL source (J0800.6 - 4806, of $\sim 0.3^{\circ}$ semimajor axis) is $1.7^{\circ}$ in the plane of the sky. Pshirkov (2016) compiled Fermi LAT data taken from 2008 August 4 to 2015 July 1 . The author reduced them with the same latest routines as the third Fermi catalogue (Acero et al. 2015) and galaxy emission models (see also Werner et al. 2013, for details on Fermi LAT data reduction). No point sources were discovered, but a $6.1 \sigma$ excess of gamma-ray emission was detected. The excess covered an area of $1 \mathrm{deg}^{2}$, where the binary system WR 11 is close to the centre. The location of such excess is given in TS (test statistics) values, similar to probability contours. The maximum contour of 37 TS $(=6.1 \sigma)$ extends in a circle of 3 arcmin diameter, and encompasses WR 11 (see Figure 3 of Pshirkov 2016).

A search of the Simbad database in a 15 arcmin radius circle centred at WR 11 resulted in $\sim 470$ objects, including Young Stellar Objects and protostellar candidates $(\sim 200)$, stars $(\sim 160)$, and X-ray sources $(\sim 60)$. WR 11 belongs to the Vela OB2 association that gathers a hundred early-type candidate members (de Zeeuw et al. 1999). Pozzo et al. (2000) identified an association of low-mass pre-main sequence stars surrounding WR 11, showing strong X-ray emission. Many of the stars are assembled in the Gamma Vel cluster (Jeffries et al. 2014). A Spitzer survey for circumstellar material around the low-mass association members reported by Hernández et al. (2008) revealed a low disc frequency.

\section{THE FIELD OF WR 11 AT RADIO CONTINUUM}

I searched the Australia Telescope Online Archive (ATOA, www.atnf.csiro.au/atoa) for data at the lowest frequency bands, $L(1.4 \mathrm{GHz})$ and $S(2.5 \mathrm{GHz})$, where NT (synchrotron) emission should be stronger ${ }^{2}$. The field of WR 11 was observed from 1999 to 2001, at various array configurations including $6^{*}, 375,1.5^{*}, 750^{*}$, and EW352 (Project C787), often during LST ranges of a few hours. Raw data taken along 15 different days from June 12 to 2001 December 12 provided enough sensitivity and resolution to detect WR 11 and were reduced for the present study. The configuration set comprised baselines between 30 and $6000 \mathrm{~m}$; the sizes of the largest well-imaged structures were 8 and $13 \mathrm{arcmin}$ at 2.5 and $1.4 \mathrm{GHz}^{3}$. The observing bands, of $128 \mathrm{MHz}$ bandwidths, were centred at $1.384 \mathrm{MHz}$ and $2.496 \mathrm{MHz}$. The FoVs were 33 and 20 arcmin, respectively. The total time on source was $\sim 12 \mathrm{~h}$ at each band. The source 1934-638 served as flux calibrator and the source $0823-500$ was monitored as phase calibrator ${ }^{4}$.

\footnotetext{
${ }^{2}$ Analysis of archive data at higher frequencies will be given elsewhere.

${ }^{3}$ http://www.narrabri.atnf.csiro.au/observing/users_guide/users guide.html

${ }^{4}$ The archive data used correspond to the ATCA configurations $6 \mathrm{~A}, 6 \mathrm{~B}, 6 \mathrm{D}$, 1.5A, 1.5D, 750D, 375, and EW352. The observing dates were June 6, June 14 , July 1, July 4, July 31, August 2, August 24, August 28, September 5 ,
} 

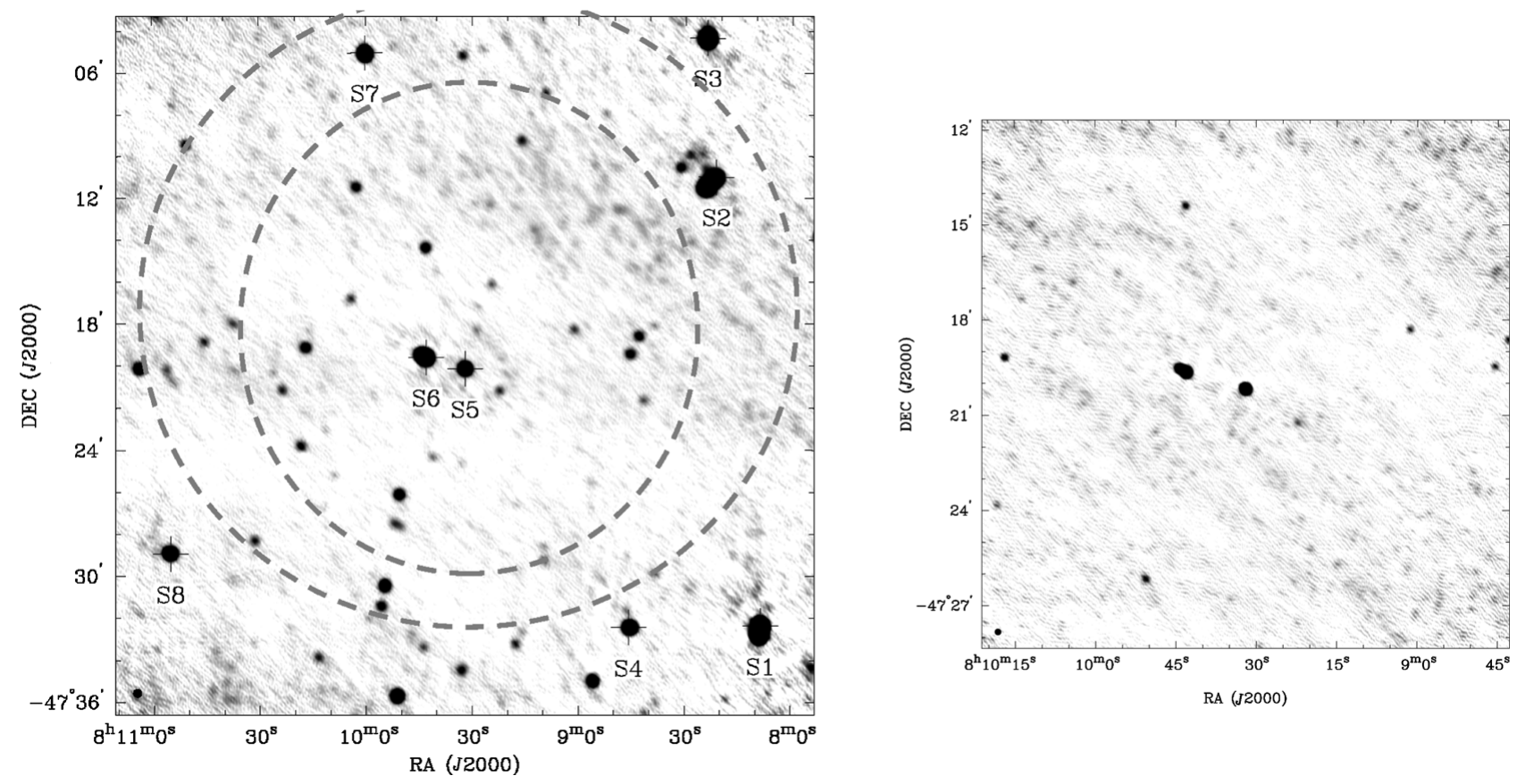

Figure 1. Radio continuum emission at $1.384 \mathrm{GHz}$ (left) and $2.496 \mathrm{GHz}$ (right), central regions; ATOA data (project C787). The rms of each map is $\leq 0.2 \mathrm{mJy}_{\text {beam }}^{-1}$. The synthesised beams are $24 \operatorname{arcsec} \times 24 \operatorname{arcsec}$ and $12 \operatorname{arcsec} \times 12$ arcsec. The brightest sources are marked with crosses and named S1 to S8 (see also Table 1). The dashed circles in the left image represent the Test Statistics (TS) at 20 (outer) and 25 (inner) of the Fermi LAT excess (see Pshirkov 2016: Figure 2 and Werner et al. 2013 for details).

The MIRIAD package was used for data reduction, which was performed in the standard way. The best images were obtained with robust weighting, and a rms near the theoretical one at each band was attained. At the image centre, the resulting rms remained below $0.1 \mathrm{mJy}^{\text {beam }}{ }^{-1}$ at each band. The synthesised beams configured at highest resolution were 11.8 arcsec $\times 2.7 \operatorname{arcsec}, P A=45.9^{\circ}(S$ band) and $22.6 \operatorname{arcsec} \times 5.8 \operatorname{arcsec}, P A=43.6^{\circ}$ ( $L$ band). The beams are very elongated because the $L S T$ ranges at most of the configurations were short ( 2 to $3 \mathrm{~h}$ ), since the primary goal of project $\mathrm{C} 787$ was not to give the best image of the field, but to focus on WR 11. The highest-resolution images were convolved with circular beams of $24 \operatorname{arcsec} \times 24 \operatorname{arcsec}(L$ band) and $12 \operatorname{arcsec} \times 12 \operatorname{arcsec}(S$ band) (Figure 1$)$.

The area to analyse was constrained by and thus circumscribed to the extension of the radio image at the larger frequency (given by its primary beam), but it agreed also with the central region of the Fermi LAT excess reported by Pshirkov (2016); see Figure 1, left.

The ATCA data revealed no extended emission. The $L$ band image showed many more sources than the $S$-band one, at the same sky area. The search for HE counterparts was focussed on the study of the stronger sources at $L$ band. We selected the objects detected with an integrated flux of $10 \mathrm{mJy}$ or above. These values are of the order of those usually quoted for radio-gamma SED fits (e.g. del Valle \& Romero 2012). Eight sources fulfilled the mentioned condition. They are

September 22, October 13, November 3, November 26, and 2001 December 12. listed in Table 1, and labelled S1 to S8, in order of increasing right ascension.

S3, S5 and S7 are discrete sources at both bands ${ }^{5}$. S1, S4 and S6, two-peaked, appeared as double sources. S8 is slightly elongated to the east, and S2 presents some substructure.

The integrated fluxes were derived either by fitting Gaussians to each discrete source or by measuring the flux above the $3 \sigma$ contour $(1 \sigma=1 \mathrm{rms})$, in the higher resolution images. In the latter case, the errors quoted in the flux densities correspond to the difference between the flux above $3 \sigma$ and that above $2 \sigma$.

Spectral indices were computed from the integrated flux at $1.4 \mathrm{GHz}$ and $2.5 \mathrm{GHz}$ for each source, as $\alpha=$ $\log \left(S_{2} / S_{1}\right) / \log \left(v_{2} / v_{1}\right)$, and are listed in Table 1 .

The system WR 11 had been also observed in 1998 February (C599) at $S$ and $L$ bands, at configuration 6A, 128-MHz bandwidths and same calibrators as for $\mathrm{C} 787$ data. I reduced the raw data corresponding to $15 \mathrm{~h}$ on source taken during day 27 , to obtain images with the highest angular resolution possible with the ATCA and as an additional check for flux measurements and spectral index values. The synthesised beams, with robust weighting, resulted in $10 \operatorname{arcsec} \times 7$ arc$\sec (L$ band) and $5 \operatorname{arcsec} \times 3 \operatorname{arcsec}(S$ band), and the rms equal to $0.06 \mathrm{mJy}^{\text {beam }}{ }^{-1}$.

\footnotetext{
${ }^{5}$ The deconvolved major and minor axis after a Gaussian fit resulted $7.1 \operatorname{arcsec} \times 4.7 \operatorname{arcsec}$ for $\mathrm{S} 3,4.1 \operatorname{arcsec} \times 1.7 \operatorname{arcsec}$ for S5, and $7.3 \operatorname{arcsec} \times 2.5 \operatorname{arcsec}$ for $\mathrm{S} 7$.
} 
Table 1. Radio fluxes of the main sources in the field of WR 11.

\begin{tabular}{|c|c|c|c|c|c|c|c|c|}
\hline ID & $\begin{array}{l}\mathrm{RA}(\mathrm{J} 2000) \\
(\mathrm{hms})\end{array}$ & $\begin{array}{l}\operatorname{Dec}(\mathrm{J} 2000) \\
(\mathrm{dms})\end{array}$ & $\begin{array}{l}S_{1.4 \mathrm{GHz}} \\
(\mathrm{mJy})\end{array}$ & $\underset{(\mathrm{mJy})}{S_{2.5 \mathrm{GHz}}}$ & $\alpha$ & $\begin{array}{l}\text { Simbad or NED } \\
\text { counterparts }\end{array}$ & Ref. & Notes \\
\hline S1 & 08:08:08.409 & $-47: 32: 23.08$ & $140 \pm 5$ & $100 \pm 4$ & $-0.6 \pm 0.14$ & $\begin{array}{c}\text { GES J08080881-4732336 } \\
\text { GES J08080943-4732250 } \\
\text { HHC2008-78 }\end{array}$ & $\begin{array}{l}\text { (a) } \\
\text { (a) } \\
\text { (b) }\end{array}$ & Proplyd \\
\hline S2 & 08:08:21.330 & $-47: 11: 00.63$ & $64 \pm 2$ & $18 \pm 3$ & $-2.1 \pm 0.4$ & HHC2008-106 & (b) & Proplyd \\
\hline S3 & $08: 08: 23.827$ & $-47: 04: 21.48$ & $77 \pm 2$ & $70 \pm 4$ & $-0.15 \pm 0.15$ & & & $?$ \\
\hline S4 & 08:08:45.737 & $-47: 32: 29.48$ & $12 \pm 1$ & $4 \pm 1$ & $-1.8 \pm 0.7$ & & & $?$ \\
\hline S5 & 08:09:31.955 & $-47: 20: 11.80$ & $11.0 \pm 0.5$ & $18.0 \pm 0.5$ & $+0.85 \pm 0.15$ & WR 11 & & WR 11 \\
\hline S6 & 08:09:42.971 & $-47: 19: 39.74$ & $43 \pm 2$ & $25 \pm 2$ & $-0.9 \pm 0.25$ & $\begin{array}{c}\text { MOST0808-471 } \\
\text { 2XMM J080942.1-471952 } \\
\text { 2MASS J08094219-4719526 }\end{array}$ & $\begin{array}{l}\text { (c) } \\
\text { (d) } \\
\text { (e) }\end{array}$ & $?$ \\
\hline S7 & 08:10:00.152 & $-47: 05: 05.60$ & $19 \pm 1$ & $11.5 \pm 1$ & $-0.85 \pm 0.3$ & $\begin{array}{c}\text { IRAS 08084-4656 } \\
\text { 2MASX J08100010-4705059 }\end{array}$ & $\begin{array}{l}\text { (f) } \\
\text { (e) }\end{array}$ & R.Galaxy \\
\hline $\mathrm{S} 8$ & $08: 10: 55.213$ & $-47: 28: 57.68$ & $9 \pm 1$ & $<3$ & $<-1.5$ & & & $?$ \\
\hline
\end{tabular}

Strongest sources at 1.4 GHz. ID: name given here, coordinates, fluxes measured at $L$ and $S$ bands (see Section 3), corresponding spectral index $\alpha\left(S_{v} \propto \nu^{\alpha}\right)$, objects from web databases overlapping the sources S1 to S8 together with their references, and most possible counterpart according to this work. (a): Jeffries et al. (2014), (b): Hernández et al. (2008), (c) Jones (1985), (d): Watson et al. (2009), (e): Skrutskie et al. (2006), (f): Helou \& Walker (1988). Deconvolved major and minor axis from Gaussian fits are $7.1 \operatorname{arcsrc} \times 4.7 \operatorname{arcsec}$ for S3, $4.1 \operatorname{arcsec} \times 1.7 \operatorname{arcsec}$ for S5, and $7.3 \operatorname{arcsec} \times 2.5 \operatorname{arcsec}$ for S7.

The WR 11 field characterisation was completed with the survey of the sources above three times the larger noise at the $L$-band image $\left(\sigma=0.2 \mathrm{mJy}\right.$ beam $\left.^{-1}\right)$. Table 2 lists all the sources-discrete and point-like-discovered in that way. Their fluxes were obtained by Gaussian fitting and the Simbad database was searched for possible counterpart identifications (see Table 2).

\section{RESULTS}

Helped with Simbad and NED databases, we carried out a search of objects in 4-arcmin radius circles around sources $\mathrm{S} 1$ to $\mathrm{S} 8$, to seek information about their nature. Such search radius granted us to collect sources from web lists at the different spectral ranges, and not only the recent most accurate ones; in the case of an extended source-counterpart, we sought its extension in the literature. Figures 2 and 3 display the individual images of S1 to S8 at maximum angular resolution (data from project C599) at one frequency; the objects found at the web databases in each sub-field are also represented.

The source S1 appears as double at both observing bands. Its spectral index is negative $(-0.6 \pm 0.14)$. Hernández et al. (2008) listed three Young Stellar Objects superposed to S1, and named them as \#74 (southern one), \#78 (central one), and \#81 (northern one), see Table 1 and Figure 2. According to IR (Spitzer) colours, the objects 74 and 81 are characterised as diskless; and the central object, \#78, corresponds to a proplyd: an evolved disk of a low-mass star (K5 or later). The linear extension of S1, if member of the gamma Vel cluster at $370 \mathrm{pc}$, is $\sim 20000 \mathrm{AU}$. The shape of S1 agrees with a central object and two outflows, and the size is alike the proplyds with negative spectral index detected by Mücke et al. (2002), supporting the association of \#78 (hereafter HHC2008-78) with S1.

The source S2 shows three maxima at both frequencies, and an average spectral index $\alpha$ of $-2.1 \pm 0.4$. The size of $\mathrm{S} 2$, at $370 \mathrm{pc}$, is $30000 \mathrm{AU}$. The object \#106 from Hernández et al. (2008), hereafter HHC2008-106, superposed to S2, is associated with a proplyd of a star with photometric colours between $\mathrm{K} 5$ and $\mathrm{M} 0$ and identified as an optically thick disk.

The source S3 has no counterpart at the consulted databases and a negative spectral index, close to 0 .

The source S4 presents a negative spectral index $(-1.8 \pm$ $0.7)$ and appears as double, with no counterpart in the literature.

The system WR 11 is located at the centre of S5. The Gamma Vel cluster, formed by low mass stars and protostars (Jeffries et al. 2014), extends around S5. The stars GES J08092860-4720178 and gamma ${ }^{1}$ Vel are nearby but detached from S5. The spectral index of this source is $+0.85 \pm 0.15$, as expected from thermal stellar wind(s). The radio source was detected in the AT20G survey (www.atnf.csiro.au/research/AT20G/) at 5, 8, and $19.9 \mathrm{GHz}$, with fluxes of $27 \pm 2,50 \pm 3$, and $86 \pm 4 \mathrm{mJy}$. The corresponding spectral indices are 1.3 (between 5 and $8 \mathrm{GHz}$ ), and 0.6 (between 8 and $19.9 \mathrm{GHz}$ ). Jones (1985) observed the system WR 11 with MOST (band centred at $843 \mathrm{MHz}$ ) and measured a flux of $8.2 \pm 1.0 \mathrm{mJy}$. The spectral index between $843 \mathrm{MHz}$ and $1.4 \mathrm{GHz}$ is $0.6 \pm 0.3$.

The source S6 is a double, and 2 arcmin away from WR 11. Its spectral index is negative $(-0.9 \pm 0.25)$ and three objects are superposed to it. One is a MOST discrete source, MOST0808-471, with a flux of $69 \pm 2$ mJy (Jones 1985). The other two, catalogued as an X-ray and an IR sources have the same coordinates, and thus could be the same object. Hernández et al. (2008) classify the IR source as a diskless 
Table 2. Sources detected at the 1384-MHz image central region.

\begin{tabular}{|c|c|c|c|c|c|c|}
\hline \# & $\begin{array}{l}\text { RA (J2000) } \\
\quad(\mathrm{hms})\end{array}$ & $\begin{array}{l}\operatorname{Dec}(\mathrm{J} 2000) \\
\quad(\mathrm{dms})\end{array}$ & $\begin{array}{c}S_{1.4 \mathrm{GHz}} \\
(\mathrm{mJy})\end{array}$ & $\begin{array}{l}\text { Deconvolved } \\
\text { axes (",,") }\end{array}$ & Simbad & Notes \\
\hline 1 & 08:07:53.841 & $-47: 34: 22.162$ & $2.2 \pm 0.2$ & & - & point-like \\
\hline 2 & 08:08:08.572 & $-47: 32: 33.677$ & $145 \pm 15$ & $36.2,6.1$ & $\mathrm{Y}^{*} ?$ & discrete \\
\hline 3 & 08:08:23.778 & $-47: 04: 21.643$ & $79 \pm 2$ & $9.0,5.0$ & - & discrete \\
\hline 4 & 08:08:25.195 & $-47: 09: 53.526$ & $1.0 \pm 0.1$ & $20.1,10.6$ & - & discrete \\
\hline 5 & 08:08:28.339 & $-47: 09: 56.829$ & $1.5 \pm 0.1$ & $23.0,12.7$ & $\mathrm{Y}^{*} ?$ & discrete \\
\hline 6 & 08:08:31.154 & $-47: 10: 31.793$ & $1.9 \pm 0.1$ & $12.9,7.5$ & - & discrete \\
\hline 7 & 08:08:42.936 & $-47: 18: 37.185$ & $1.7 \pm 0.1$ & & - & point-like \\
\hline 8 & 08:08:45.357 & $-47: 19: 28.579$ & $1.8 \pm 0.1$ & $9.6,2.5$ & $\mathrm{XMM}^{*}$ & discrete \\
\hline 9 & 08:08:45.389 & $-47: 32: 29.906$ & $12 \pm 0.5$ & $10.7,5.8$ & - & discrete \\
\hline 10 & 08:08:55.876 & $-47: 35: 03.080$ & $5.0 \pm 0.5$ & & $\mathrm{Y}^{*} ?$ & point-like \\
\hline 11 & 08:09:01.145 & $-47: 18: 18.947$ & $0.8 \pm 0.1$ & & - & point-like \\
\hline 12 & 08:09:15.986 & $-47: 09: 17.010$ & $1.2 \pm 0.1$ & $13.2,8.4$ & - & discrete \\
\hline 13 & 08:09:31.956 & $-47: 20: 11.610$ & $11.3 \pm 0.1$ & $6.2,3.0$ & WR 11 & discrete \\
\hline 14 & 08:09:32.971 & $-47: 34: 32.881$ & $1.5 \pm 0.1$ & $14.0,9.7$ & - & discrete \\
\hline 15 & 08:09:43.112 & $-47: 14: 24.504$ & $2.0 \pm 0.1$ & & - & point-like \\
\hline 16 & 08:09:43.318 & $-47: 19: 38.027$ & $43.0 \pm 0.1$ & $16.2,2.8$ & MOST & discrete \\
\hline 17 & 08:09:43.748 & $-47: 33: 27.319$ & $0.7 \pm 0.1$ & & $\mathrm{Y}^{*} ?$ & point-like \\
\hline 18 & 08:09:50.561 & $-47: 26: 10.844$ & $2.7 \pm 0.2$ & & pr? & point-like \\
\hline 19 & 08:09:51.178 & $-47: 35: 46.938$ & $7.3 \pm 0.1$ & $7.9,5.6$ & $\mathrm{Y}^{*} ?$ & discrete \\
\hline 20 & 08:09:51.306 & $-47: 27: 36.077$ & $1.4 \pm 0.1$ & & $\mathrm{XMM}^{*}$ & point-like \\
\hline 21 & 08:09:54.667 & $-47: 30: 31.099$ & $3.9 \pm 0.3$ & $9.4,1.5$ & - & discrete \\
\hline 22 & 08:09:55.642 & $-47: 31: 29.674$ & $1.7 \pm 0.1$ & & - & point-like \\
\hline 23 & 08:10:00.124 & $-47: 05: 06.390$ & $19.0 \pm 1.0$ & $8.7,1.5$ & IRAS & discrete \\
\hline 24 & 08:10:02.680 & $-47: 11: 30.112$ & $1.7 \pm 0.1$ & & - & point-like \\
\hline 25 & 08:10:13.332 & $-47: 33: 55.950$ & $1.0 \pm 0.1$ & & - & point-like \\
\hline 26 & 08:10:16.947 & $-47: 19: 10.242$ & $2.3 \pm 0.1$ & $9.5,3.2$ & - & discrete \\
\hline 27 & $08: 10: 18.286$ & $-47: 23: 50.957$ & $1.3 \pm 0.1$ & & - & point-like \\
\hline 28 & $08: 10: 23.525$ & $-47: 21: 11.805$ & $1.0 \pm 0.1$ & & $\mathrm{XMM}^{*}$ & point-like \\
\hline 29 & $08: 10: 31.408$ & $-47: 28: 21.710$ & $1.0 \pm 0.1$ & & - & point-like \\
\hline 30 & $08: 10: 37.417$ & $-47: 18: 00.427$ & $1.0 \pm 0.1$ & & - & point-like \\
\hline 31 & $08: 10: 45.544$ & $-47: 18: 52.399$ & $1.0 \pm 0.1$ & & - & point-like \\
\hline 32 & $08: 10: 50.596$ & $-47: 09: 26.975$ & $1.4 \pm 0.1$ & & - & point-like \\
\hline 33 & $08: 10: 55.318$ & $-47: 28: 56.504$ & $9.9 \pm 0.5$ & $12.3,7.1$ & - & discrete \\
\hline 34 & $08: 10: 56.121$ & $-47: 20: 12.757$ & $0.9 \pm 0.1$ & $16.1,6.3$ & $\mathrm{XMM}^{*}$ & discrete \\
\hline 35 & 08:11:03.867 & $-47: 20: 07.978$ & $3.8 \pm 0.3$ & $8.1,3.4$ & - & discrete \\
\hline
\end{tabular}

$\mathrm{Y}^{*}$ ?: young stellar object candidate; $\mathrm{XMM}^{*}: 2 \mathrm{XMM}$ star; MOST: MOST source; pr?: pre-main sequence star candidate, IRAS: IRAS source. Source \#3 is S3, source \#13 is S5 and source \#23 is S7, see Table 1.

YSO (\#158). The spectral index of S6 between $843 \mathrm{MHz}$ and $1.4 \mathrm{GHz}$ is $-0.95 \pm 0.15$.

The source S7 correlates with the source IRAS 080484-4656, and the double galaxy 2MASX J08100010-4705059, $0.17 \times 0.17 \operatorname{arcmin}^{2}$ in size (Skrutskie et al. 2006, 2MASS). S7 shows a negative spectral index $(-0.9 \pm 0.3)$.

Source S8 has no counterparts neither in NED nor in the Simbad databases. Its spectral index is less than -1.5 .

\section{DISCUSSION}

The search for counterparts of the radio sources detected in this work resulted in possible associations of S1 and S2 with YSO proplyds, S5 with the system WR 11, and S7 with a double galaxy. S6, at 2 arcmin from WR 11, is a double source with NT radiation. S4 is alike but weaker and at $\sim 16$ arcmin from WR 11. All detected radio sources but S5 show negative spectral indices.

The simplest hypothesis is that a single source is responsible of the excess emission detected with Fermi LAT data. Under such assumption, the two possible scenarios to analyse are whether WR 11, or another source in the field, are the radio counterpart of the Fermi source.

\subsection{Sources other than WR 11 as the Fermi LAT counterparts}

The positional coincidence between the source S1 and the object HHC2008-78, and the fact that other proplyds have been detected as radio sources at low frequencies (Masqué, Dzib, \& Rodríguez 2014, and references therein) favours the identification of S1 with a proplyd. The same can be said of S2 and HHC2008-106. Proplyds were detected at more than one frequency, and some of them show a spectral index 

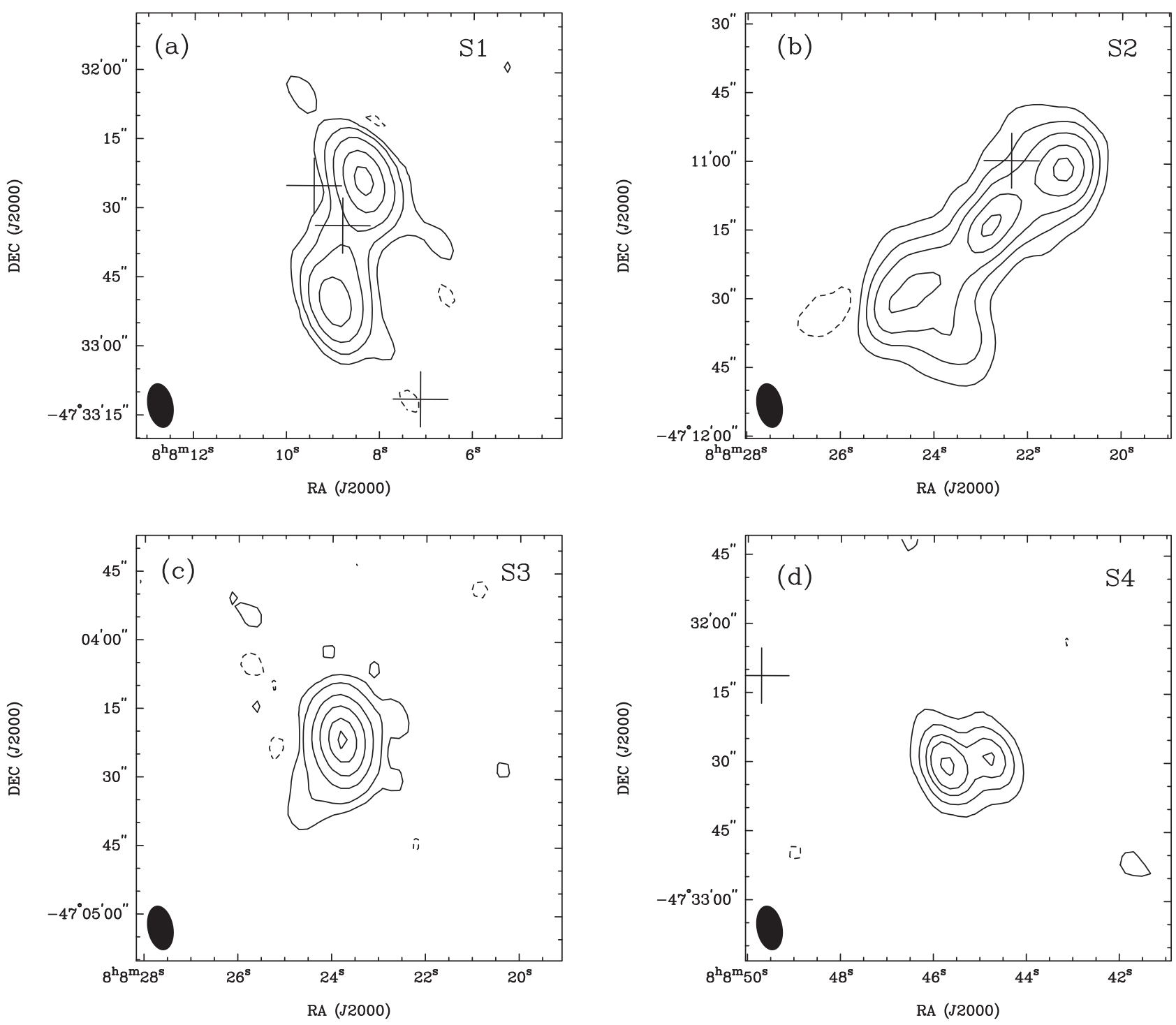

Figure 2. Zoom on sources S1 to S4 (images from C599 data at $1.4 \mathrm{GHz}$ ). Coordinates: RA, Dec (J2000). Crosses: YSO or protostellar candidates; small boxes: X-ray sources. Black circles: stars. (a): S1; contours $-3,3,10,30,90$, and 200 in units of $\sigma=0.2 \mathrm{mJy} \mathrm{beam}^{-1}$. (b): S2; contours -3 , $3,10,20,35$, and 50 in units of $\sigma=0.1 \mathrm{mJy}_{\text {beam }}{ }^{-1}$. (c): S3; contours $-3,3,10,40,100,200$, and 300 in units of $\sigma=0.15 \mathrm{mJy}$ beam ${ }^{-1}$. (d): S4; contours $-3,3,10,20,30$, and 50 in units of $\sigma=0.09 \mathrm{mJy}_{\text {beam }}{ }^{-1}$.

different from thermal (e.g. Mücke et al. 2002). However, none has been identified with (Fermi) gamma-ray sources (Acero et al. 2015; Armitage 2015).

The 2MASS catalogue of extended sources (XSC in Skrutskie et al. 2006) suggests that 2MASX J08100010-4705059 is a double-lobed galaxy, based on the emission at $J, K$, and $H_{\mathrm{s}}$ bands. The object would also be detected as the source IRAS 08084-4656. They are positionally coincident with the radio source S7. There are many Fermi LAT sources identified with radio galaxies, with gamma-ray fluxes of the order of the one detected by Pshirkov (2016). We note that while the gamma ray fluxes have the same magnitude, the radio flux densities of this source is much lower than those of the Fermi confirmed radio galaxy associations. Unfortunately, since S7 is a discrete source, it is not possible to derive constraints from the radio data presented here.

The source S6 is double, extended along $\sim 45$ arcsec. If it is a galactic object (for instance, a microquasar), it should be detected as a stellar source too. The shape and the spectral index are consistent with those of a radio galaxy. Such object, for instance if 30-kpc long, will be at a distance of $100 \mathrm{Mpc}$, like other typical FRI or FRII galaxies. The Fermi LAT energy flux of $1.8 \pm 0.6 \times 10^{9} \mathrm{erg} \mathrm{cm}^{-2} \mathrm{~s}^{-1}$ quoted by Pshirkov (2016) implies, at $100 \mathrm{Mpc}$, a luminosity of $\sim 10^{42} \mathrm{erg} \mathrm{s}^{-1}$.

A radio emission spectral index of $\alpha=-0.9$ yields an electron energy distribution power law of index $p=1-$ $2 \alpha=2.8$, and thus a photon spectral index $\Gamma \sim(p+1) / 2=$ 1.8 (Vila \& Aharonian 2009). The flux measured here 

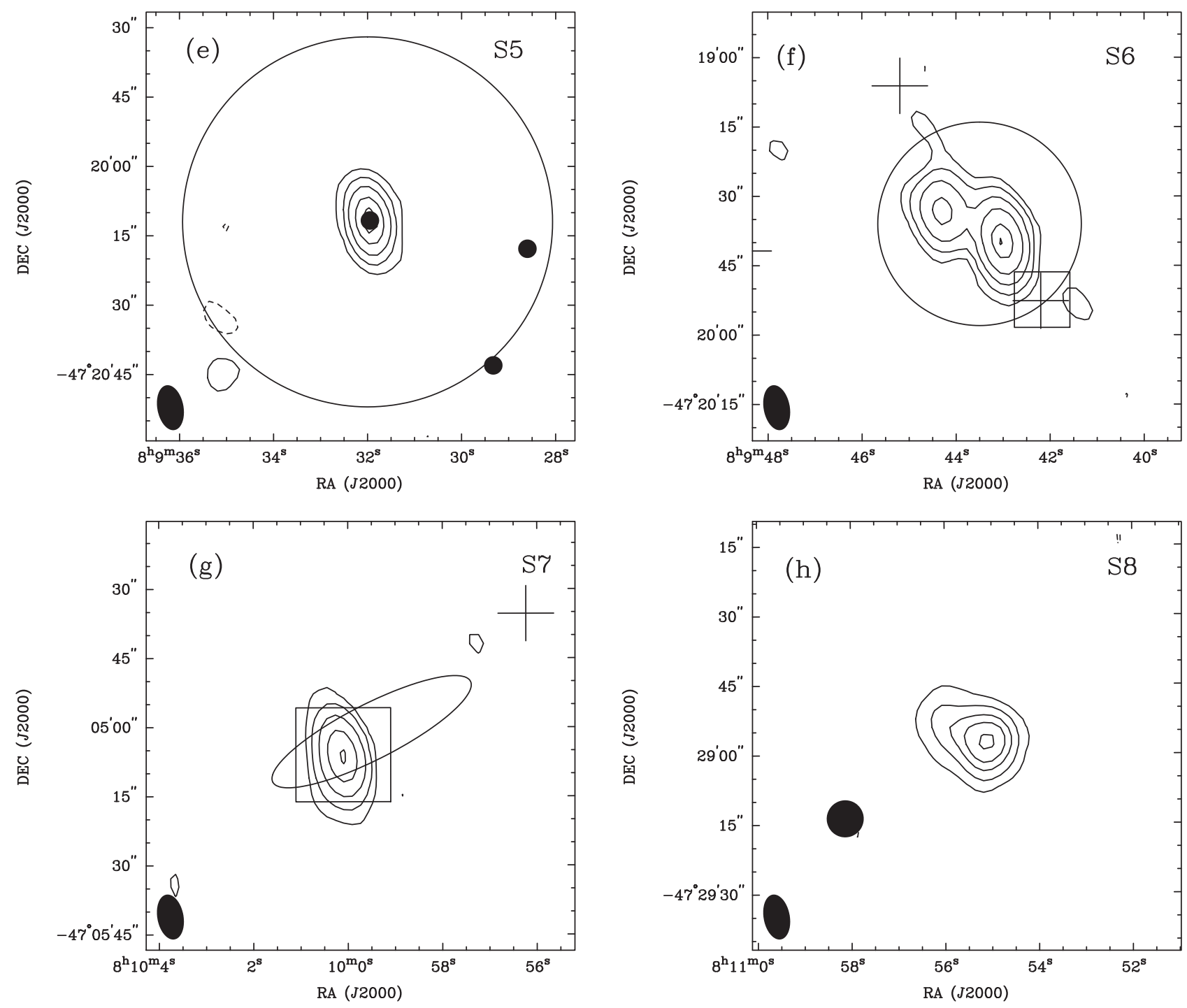

Figure 3. Same as Figure 2 on sources S5 to S8 (images from C599 data at $1.4 \mathrm{GHz}$ ). (e): S5; contours $-3,3,10,25,55$, and 90 in units of $\sigma=0.09$ mJy beam ${ }^{-1}$. The circle represents the centre of the gamma Vel cluster. (f): S6; contours $-3,3,10,30,80,200$, and 280 in units of $\sigma=0.09 \mathrm{mJy}$ beam $^{-1}$. (g): S7; contours $-3,3,10,40,80$, and 130 in units of $\sigma=0.09$ mJy beam $^{-1}$. The ellipse marks the IRAS source and the box represents the radio galaxy, see Table 1. (h): S8; contours $-3,3,10,20,30$, and 43 in units of $\sigma=0.09$ mJy beam $^{-1}$.

corresponds to the extended emission. For most gamma-ray radio galaxies, the major contribution to the gamma-ray emission comes from their inner jets (luminosities and gamma spectral indices of FRI and II found from Fermi LAT data are given in Grandi 2012, her Figure 1). S6 could be a radio galaxy, that radiates the flux detected by Fermi LAT. And S6 is, as WR 11, inside the maximum probability contour of the Fermi LAT source position.

\subsection{WR 11 as the Fermi LAT counterpart}

The detection of a MOST source (Jones 1985) at the position of WR 11 allows to derive an average spectral index of +0.6 from 0.843 to $1.4 \mathrm{GHz}$, and similar values up to $20 \mathrm{GHz}$. That NT emission is only present below $843 \mathrm{MHz}$ seems unlikely (Reitberger et al. 2014, 2015). However, absence of
NT radio emission is not an argument to reject the hypothesis of a potential association between WR 11 and the Fermi LAT source. A very low magnetic field or too short energy-loss timescales for electrons would inhibit synchrotron emission to be detected. This emission could be affected by a strong free-free absorption from a dense circumstellar matter, a small orbit size and/or a complex geometry of the system, too.

The star Eta Carinae (HD 93308) shares some features with WR 11. The wind kinetic powers are of the same order (2.8 and $1.6 \times 10^{37} \mathrm{erg} \mathrm{s}^{-1}$, respectively, De Becker \& Raucq 2013, and references therein). The former is a LVB star with a massive unseen companion (Damineli et al. 2008), thus considered a CWB. Its radio emission at high-angular resolution was measured since the first times of the ATCA. Duncan \& White (2003) (see also White et al. 2005) pictured 
the radio source at $3-\mathrm{cm}$ wavelength as variable between two states: one up to 4 arcmin in extent, and lower flux $(\sim 1 \mathrm{mJy})$ at the apastron, and another of a discrete source (extension less than $1 \mathrm{arcsec}$ ) and stronger ( 2 to $3 \mathrm{mJy}$ ) near periastron. The variability correlates with the binary period. The authors interpreted that the orbit of the companion is within an extended and dense medium that is being released by, and largely circumvents, the LBV. There is no signature that the variability is due to NT radio radiation.

Since Eta Car displays gamma-ray emission (Tavani et al. 2009; Abdo et al. 2010) and NT X-ray emission (Sekiguchi et al. 2009), it was included in the PACWBs catalogue (De Becker \& Raucq 2013). Very recently, Ohm et al. (2015) presented a time-dependent model of the HE emission in Eta Car. The model achieved a good agreement with the stellar lightcurve. They found that the measured spectrum can result from accelerated protons that interact with the dense LBV wind: no emission coming from primary electrons is further needed. The gamma-ray detectability with current instruments in $\mathrm{Ohm}$ et al. model depends strongly on the stellar mass loss rates.

WR 11 is the second CWB with no conclusive signs of NT emission. Its mass loss rate is less than 10 times that of Eta Car (see De Becker \& Raucq 2013, and references therein); the primary is a WR star instead of a LBV. It was mapped as a discrete source so far (less than 1 arcsec), with no circumstellar emission. A model tailored to this system is crucial to find whether the WR stellar wind is capable of absorbing the radio NT radiation in gamma ${ }^{2}$ Velorum, and which are the processes that could give rise to the $\mathrm{GeV}$ excess of emission.

\section{SUMMARY}

Telescope data archives are a profitable source of unpublished results. The reduction and analysis of archival (ATCA) radio interferometric raw data of the field of WR 11 at 1.4 and 2.5 $\mathrm{GHz}$ led to the following findings.

1. There are tens of radio sources in the field of WR 11, unclassified and uncatalogued. One disclosed in this work and labelled S6, in principle, is a candidate to be investigated as a gamma-ray producer.

2. The systems WR 11 and Eta Car have some similarities but the differences-especially the mass loss rate values and the presence or absence of dense circumstellar matter-preclude the use of same frameworks.

3. The emission of WR 11 from frequencies above 843 $\mathrm{MHz}$, up to $20 \mathrm{GHz}$, presents thermal spectral indices.

4. The absence of NT emission is not enough to discard WR 11 as the one producing the Fermi LAT emission excess.

Due to sensitivity issues, the 7-yr Fermi data at the position of WR 11 cannot be used to search variability that could be locked to the system phase, for instance. But the radio sources in the field can be investigated in more detail, by carrying out observations at very low frequencies and arcsec resolution. The Giant Metrewave Radio Telescope is the best suited to that aim. Radio flux variability studies of the field sources could give a hint on their nature. Together with these observational results, a model to explain the emission of WR 11 along the entire electromagnetic spectrum is needed.

\section{ACKNOWLEDGEMENTS}

The author thanks an anonymous referee, whose comments and suggestions resulted in improving the article. PB is grateful to $\mathrm{M}$. Fernández López, M. De Becker, S. M. Dougherty and S. del Palacio. This research has made use of the SIMBAD database, operated at CDS, Strasbourg, France, and of the NASA/IPAC Extragalactic Database (NED) which is operated by the Jet Propulsion Laboratory, California Institute of Technology, under contract with the National Aeronautics and Space Administration. This work was supported by the FONCyT project PICT 2012-878.

\section{REFERENCES}

Abdo, A. A., et al. 2010, ApJ, 723, 649

Acero, F., et al. 2015, ApJS, 218, 23

Armitage, P. J. 2015, preprint (arXiv:1509.06382)

Benaglia, P., \& Romero, G. E. 2003, A\&A, 399, 1121

Benaglia, P., Romero, G. E., Stevens, I. R., \& Torres, D. F. 2001, A\&A, 366, 605

Chapman, J. M., Leitherer, C., Koribalski, B., Bouter, R., \& Storey, M. 1999, ApJ, 518, 890

Damineli, A., et al. 2008, MNRAS, 384, 1649

De Becker, M., \& Raucq, F. 2013, A\&A, 558, A28

de la Chevrotière, A., St-Louis, N., Moffat, A. F. J., \& MiMeS Collaboration 2014, ApJ, 781, 73

de Zeeuw, P. T., Hogerwerf, R., de Bruijne, J. H. J., Brown, A. G. A., \& Blaauw, A. 1999, AJ, 117, 354

del Valle, M. V., \& Romero, G. E. 2012, A\&A, 543, A56

Dougherty, S. M., \& Williams, P. M. 2000, MNRAS, 319, 1005

Duncan, R. A., \& White, S. M. 2003, MNRAS, 338, 425

Eichler, D., \& Usov, V. 1993, ApJ, 402, 271

Grandi, P. 2012, IJMPS, 8, 25

Hartman, R. C., et al. 1999, ApJS, 123, 79

Helou, G., \& Walker, D. W., eds, 1988, Infrared astronomical satellite (IRAS) catalogs and atlases. Vol. 7, The small scale structure catalog (Washington, DC: GPO)

Hermsen, W. 1983, SSRv, 36, 61

Hernández, J., Hartmann, L., Calvet, N., Jeffries, R. D., Gutermuth, R., Muzerolle, J., \& Stauffer, J. 2008, ApJ, 686, 1195

Jeffries, R. D., et al. 2014, A\&A, 563, A94

Jones, P. A. 1985, MNRAS, 216, 613

Leitherer, C., Chapman, J. M., \& Koribalski, B. 1997, ApJ, 481, 898

Masqué, J. M., Dzib, S., \& Rodríguez, L. F. 2014, ApJ, 797, 60

Massaro, F., D’Abrusco, R., Paggi, A., Masetti, N., Giroletti, M., Tosti, G., Smith, H. A., \& Funk, S. 2013, ApJS, 209, 10

Millour, F., et al. 2007, A\&A, 464, 107

Mücke, A., Koribalski, B. S., Moffat, A. F. J., Corcoran, M. F., \& Stevens, I. R. 2002, ApJ, 571, 366

North, J. R., Tuthill, P. G., Tango, W. J., \& Davis, J. 2007, MNRAS, 377,415 
Ohm, S., Zabalza, V., Hinton, J. A., \& Parkin, E. R. 2015, MNRAS, 449, L132

Paredes, J. M., et al. 2008, A\&A, 482, 247

Pittard, J. M., Dougherty, S. M., Coker, R. F., O’Connor, E., \& Bolingbroke, N. J. 2006, A\&A, 446, 1001

Pozzo, M., Jeffries, R. D., Naylor, T., Totten, E. J., Harmer, S., \& Kenyon, M. 2000, MNRAS, 313, L23

Pshirkov, M. S. 2016, MNRAS, 457, L99

Reimer, A., \& Reimer, O. 2009, ApJ, 694, 1139

Reimer, A., Pohl, M., \& Reimer, O. 2006, ApJ, 644, 1118

Reitberger, K., Kissmann, R., Reimer, A., \& Reimer, O. 2014, ApJ, 789, 87

Reitberger, K., Kissmann, R., Reimer, A., Reimer, O., \& Dubus, G. 2014b, ApJ, 782, 96

Reitberger, K., Reimer, A., Reimer, O., \& Takahashi, H. 2015, A\&A, 577, A100
Romero, G. E., Benaglia, P., \& Torres, D. F. 1999, A\&A, 348, 868 Schild, H., et al. 2004, A\&A, 422, 177

Sekiguchi, A., Tsujimoto, M., Kitamoto, S., Ishida, M., Hamaguchi, K., Mori, H., \& Tsuboi, Y. 2009, PASJ, 61, 629

Skrutskie, M. F., et al. 2006, AJ, 131, 1163

Tavani, M., et al. 2009, ApJ, 698, L142

Vila, G. S., \& Aharonian, F. 2009, in Compact Objects and their Emission, eds. G. E. Romero \& P. Benaglia (La Plata: Paideia), ch. 1,1

Watson, M. G., et al. 2009, A\&A, 493, 339

Werner, M., Reimer, O., Reimer, A., \& Egberts, K. 2013, A\&A, 555, A102

White, S. M., Duncan, R. A., Chapman, J. M., \& Koribalski, B. 2005, in ASP Conf. Ser., Vol. 332, The Fate of the Most Massive Stars, eds. R. Humphreys \& K. Stanek (San Francisco: ASP), 129 